\title{
Knockdown of homeobox A5 by small hairpin RNA inhibits proliferation and enhances cytarabine chemosensitivity of acute myeloid leukemia cells
}

\author{
NA LI ${ }^{1 *}$, XIUHONG JIA ${ }^{1 *}$, JIANYONG WANG $^{1}$, YOUJIE LI $^{2}$ and SHUYANG XIE ${ }^{2}$ \\ ${ }^{1}$ Department of Pediatrics, Affiliated Hospital of Binzhou Medical University, Binzhou, Shandong 256003; \\ ${ }^{2}$ Department of Biochemistry and Molecular Biology, Binzhou Medical University, Yantai, Shandong 264003, P.R. China
}

Received November 14, 2014; Accepted July 31, 2015

DOI: $10.3892 / \mathrm{mmr} .2015 .4331$

\begin{abstract}
Homeobox genes encode transcription factors that are essential for embryonic morphogenesis and differentiation. Transcription factors containing the highly conserved homeobox motif show considerable promise as potential regulators of hematopoietic maturation events. Previous studies have suggested that the increased expression levels of homeobox (HOX)A genes was correlated with the cytogenetic findings associated with poor prognosis in acute myeloid leukemia and mixed lineage leukemia. The aim of the present study was to investigate the role of HOXA5 in leukemia. The U937 human leukemia cell line was transfected with a HOXA5-targeted short hairpin RNA (shRNA) to determine the effects of downregulation of the HOXA5 on proliferation, apoptosis, cell cycle distribution and chemoresistance in leukemia cells. Reverse transcription-quantitative polymerase chain reaction and western blot analyses demonstrated that the mRNA and protein expression levels of HOXA5 were markedly suppressed following transfection with an shRNA-containing vector. Knockdown of HOXA5 significantly inhibited cell proliferation, as determined by Cell Counting kit- 8 assay. Flow cytometry revealed that reduced HOXA5 expression levels resulted in cell cycle arrest at the $G_{1}$ phase, and induced
\end{abstract}

Correspondence to: Professor Xiuhong Jia, Department of Pediatrics, Affiliated Hospital of Binzhou Medical University, 661 Yellow River 2nd Road, Binzhou, Shandong 256003, P.R. China E-mail: jiaxiuhong001@163.com

*Contributed equally

Abbreviations: AML, acute myeloid leukemia; CCK-8, cell counting kit-8; GFP, green fluorescent protein; CN-AML, cytogenetically normal acute myeloid leukemia; PBS, phosphate-buffered saline; RT-qPCR, reverse transcription-quantitative polymerase chain reaction; SDS-PAGE, sodium dodecyl sulphate-polyacrylamide gel electrophoresis; shRNA, short hairpin RNA

Key words: homeobox A5, leukemia, small hairpin RNA, cell growth, apoptosis, chemosensitivity apoptosis. In addition, western blot analysis demonstrated that HOXA5 knockdown increased the expression levels of caspase-3, and reduced the expression levels of survivin in the U937 cells. Furthermore, knockdown of HOXA5 in the U937 cells enhanced their chemosensitivity to cytarabine. The results of the present study suggested that downregulation of HOXA5 by shRNA may trigger apoptosis and overcome drug resistance in leukemia cells. Therefore, HOXA5 may serve as a potential target for developing novel therapeutic strategies for leukemia.

\section{Introduction}

Acute myeloid leukemia (AML) is a hematopoietic stem cell disorder characterized by the clonal proliferation of myeloid precursors with inhibition of differentiation, leading to the accumulation of immature cells at various stages and reduction in the production of normal hematopoietic components: Erythrocytes, platelets and mature granulocytes (1). AML is the most common lethal hematological malignancy in children and young adults, and represents $3 \%$ of all cancer cases, and accounts for $\sim 257,000$ cases of cancer-associated mortality worldwide annually (2). With conventional intensive chemotherapy, the prognosis for patients with AML remains poor, with an overall long-term survival of $<30 \%$ in patients younger than 60 years old, and only $10-20 \%$ for older patients (3). Previous studies have demonstrated that therapeutic strategies targeting tumor cell growth and survival signaling pathways may provide a novel strategy to optimize AML therapy (4-6). Although numerous molecules have been identified as potential targets, only a few have a pivotal role in tumor cell proliferation and survival (7). Therefore, the identification of novel therapeutic targets and the development of novel therapeutic strategies that may effectively regulate cellular function appear to be of central importance.

Homeobox (HOX) genes comprise a large family of homeodomain-containing transcription factors, present in four separate clusters (A-D), which are key regulators of embryonic development, hematopoietic differentiation and leukemogenesis (8). Previous studies demonstrated that increased HOXA9 and Meis homeobox 1 expression were strongly associated with cytogenetically normal-AML $(9,10)$. 
HOXA5 is a member of the HOX gene family, which is known to have important roles in embryonic development and in the regulation of the differentiation process of adult cells. In addition, HOXA5 has been implicated in the differentiation of epithelial and hematopoietic cells (11). Overexpression of HOXA5 in hematopoietic progenitors results in increased granulocytic/monocytic differentiation, but reduced erythroid/megakaryocytic differentiation, which suggests that HOXA5 functions as an important regulator of hematopoietic lineage determination and maturation (12).

Studies of homeobox genes in leukemic cells provided evidence that aberrant expression may have an important role during leukemogenesis $(13,14)$. However, whether HOXA5 regulates the proliferation and apoptosis of leukemia cells, as well as the nature of the underlying mechanism, remain unknown. The aim of the present study was to investigate the potential role of HOXA5 in the development of leukemia.

\section{Materials and methods}

Cell culture. Human U937 AML cells were purchased from the Shanghai Institutes for Biological Sciences of the Chinese Academy of Sciences (Shanghai, China) and cultured in RPMI-1640 medium (GE Healthcare Life Sciences, Logan, UT, USA) supplemented with $15 \%$ fetal bovine serum (GE Healthcare Life Sciences) and 1\% antibiotics (100 IU/ml penicillin and $100 \mu \mathrm{g} / \mathrm{ml}$ streptomycin; GE Healthcare Life Sciences), at $37^{\circ} \mathrm{C}$ in a humidified atmosphere containing $5 \%$ $\mathrm{CO}_{2}$.

GFP assays. Following transfection, GFP expression in U937 cells was observed under a fluorescent microscope (Olympus DP71; Olympus, Tokyo, Japan) and then the percentage of GFP-positive cells was estimated by flow cytometry (FACS FC500; Beckman Coulter, Brea CA, USA).

In vitro transfection with shRNA. Three pairs of shRNA sequences targeting HOXA5, termed HOXA5 shRNA-1, -2, -3 , and one scramble sequence that served as a control were designed and synthesized by Shanghai GenePharma Co., Ltd. (Shanghai, China). Their target sequences are shown in Table I. The U937 cells in logarithmic growth phase were seeded into a six-well plate at a density of $5 \times 10^{5}$ cells/well and transfected using HiPerFect (Qiagen, Inc., Valencia, CA, USA) according to the manufacturer's instructions. After $48 \mathrm{~h}$, transfection efficiency was examined under the fluorescence microscope. Reverse transcription-quantitative polymerase chain reaction (RT-qPCR) and western blot analyses were performed to determine inhibitory efficacy.

$R T$ - $q P C R$. Total RNA was isolated using TRIzol ${ }^{\circledR}$ reagent (Invitrogen Life Technologies, Carlsbad, CA, USA), according to the manufacturer's instructions. A total of $2 \mu \mathrm{g}$ total RNA was reverse transcribed into cDNA. The reverse transcription reaction was performed using a PrimeScript ${ }^{\mathrm{TM}}$ RT reagent kit with gDNA Eraser (Takara Biotechnology Co., Ltd., Otsu, Japan) and RT-qPCR was performed using a SYBR Green reaction kit (Takara Biotechnology Co., Ltd.) on an ABI PRISM 7500 real-time PCR system (Applied Biosystems, Foster City, CA, USA). The reaction system of the PCR was composed of
Table I. Candidate HOXA5 shRNAs and their target sequences.

Recombinant Target sequence (5'-3')

HOXA5 ShRNA-1 GGACTACCAGTTGCATAATTA HOXA5 ShRNA-2 GCTTTCTGTTCATCTCTTTGT HOXA5 shRNA-3 GCAGAAGGAGGATTGAAATAG

HOXA5, homeobox A5; shRNA, short hairpin RNA.

SYBR green reagent, forward primer, reverse primer, template cDNA and nuclease-free distilled water. The PCR conditions were as follows: $95^{\circ} \mathrm{C}$ for $30 \mathrm{sec}, 40$ cycles of $95^{\circ} \mathrm{C}$ for $5 \mathrm{sec}$, and $60^{\circ} \mathrm{C}$ for $34 \mathrm{sec}$. The PCR primer sequences for the RT-qPCR were as follows: Forward: 5'-TGCACCACCACCTGCTTA GC-3' and reverse: 5'-GGCATGGACTGTGGTCATGAG-3' for human GAPDH; and forward: 5'-AGCCACAAATCAAGG ACACA-3' and reverse: 5'-GCTCGCTCACGGAACTATG-3' for HOXA5. qPCR for each gene of each cDNA sample was assayed in triplicate. The results were calculated using the $2^{-\Delta \Delta \mathrm{Ct}}$ method using the following equations: $\Delta \mathrm{Ct}=\mathrm{Ct}_{\text {(target gene) }}-\mathrm{Ct}_{\text {(actin) }}$; $\Delta \Delta \mathrm{Ct}=\Delta \mathrm{Ct}_{\text {(HOXA5 shRNA-treated cells) }-\Delta \mathrm{Ct} \text { (untreated control) }}$.

Western blot analysis. The cells were washed twice with cold phosphate-buffered saline (PBS) and harvested in $100 \mu \mathrm{l}$ cell lysis buffer (Nanjing KeyGEN Biotech Co., Ltd., Nanjing, China) containing protease inhibitors (Nanjing KeyGEN Biotech Co., Ltd.). A total of $50 \mu \mathrm{g}$ extracted proteins were separated on $10 \%$ SDS-PAGE (Beyotime Institute of Biotechnology, Shanghai, China), and then transferred electrophoretically onto a polyvinylidene difluoride membrane (EMD Millipore, Bedford, MA, USA). The membranes were blocked with 5\% skimmed milk for $2 \mathrm{~h}$ at room temperature, and washed three times with Tris-buffered saline with Tween-20 [TBST; $50 \mathrm{mM}$ Tris- $\mathrm{HCl}$ (pH 7.6), $150 \mathrm{mM} \mathrm{NaCl}, 0.1 \%$ Tween-20], and then incubated overnight at $4^{\circ} \mathrm{C}$ with specific antibodies. The primary antibodies used were as follows: Rabbit monoclonal anti-HOXA5 (ImmunoWay Biotechnology, Newark, DE, USA; 1:1,000 dilution; cat. no. YT2211), rabbit polyclonal anti-survivin (Wuhan Boster Biological Technology, Ltd., Wuhan, China; 1:300 dilution; cat. no. BA4055-2), rabbit polyclonal anti-caspase-3 (Wuhan Boster Biological Technology, Ltd:; 1:300 dilution; cat. no. BA2885-2) antibodies, and rabbit polyclonal anti- $\beta$-actin (Beyotime Institute of Biotechnology, Haimen, China; 1:1000 dilution; cat. no. BA2305) antibody was used as a loading control. The following day, after washing with TBST, the membrane was incubated in horseradish peroxidase-labeled goat anti-rabbit immunoglobulin G (1:5,000; cat. no. ZB-5301; OriGene Technologies, Beijing, China) for $1 \mathrm{~h}$ at room temperature. Finally, images were captured using a FluorChem FC2 gel imaging system (Alpha Innotech, San Leandro, CA, USA).

Cell proliferation assay. Cell proliferation was determined using a Cell Counting kit-8 (CCK-8) assay (Dojindo Molecular Technologies, Inc., Kumamoto, Japan). The cells were plated at a density of $1-2 \times 10^{4}$ cells/well in 96-well culture plates. Following treatment, $10 \mu \mathrm{l}$ CCK- 8 solution was added to each well and incubated for 1-4 h. The absorbance was then 


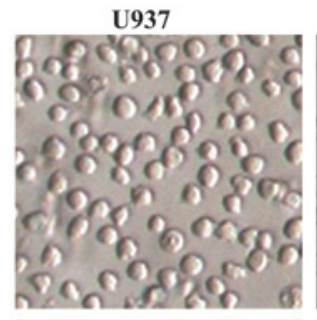

Control shRNA
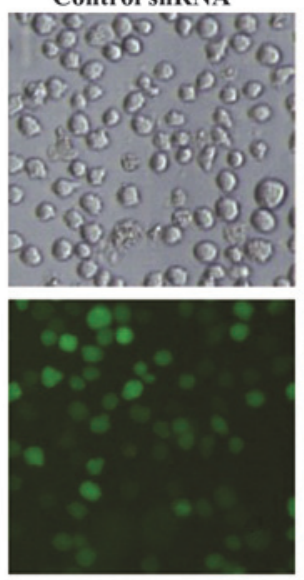

HOXA5 shRNA-3
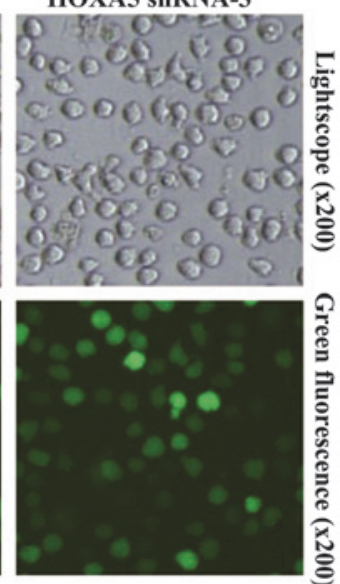

Figure 1. Transfection of HOXA5 with shRNA. Transfection efficiency was evaluated by fluorescence microscopy. The average transfection efficiency in each group was $>90 \%$. Representative images are shown. HOXA5, homeobox A5; shRNA, short hairpin RNA.

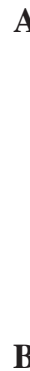

A

$\mathbf{B}$
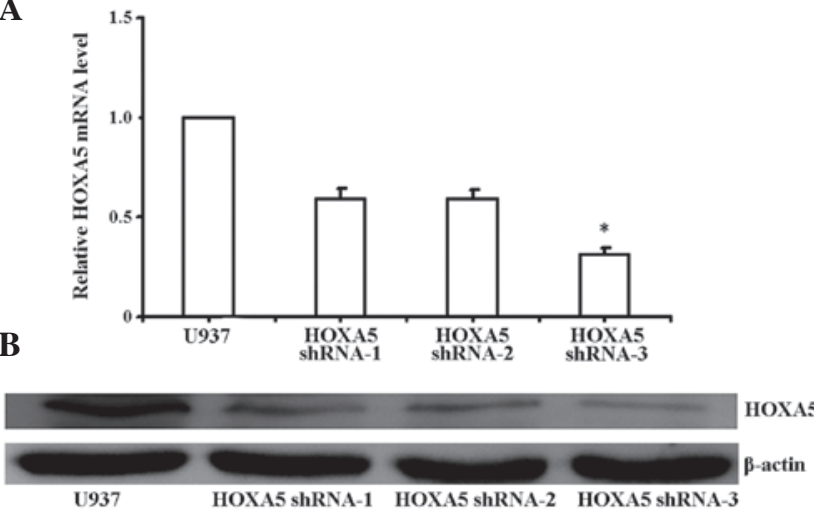

C

D
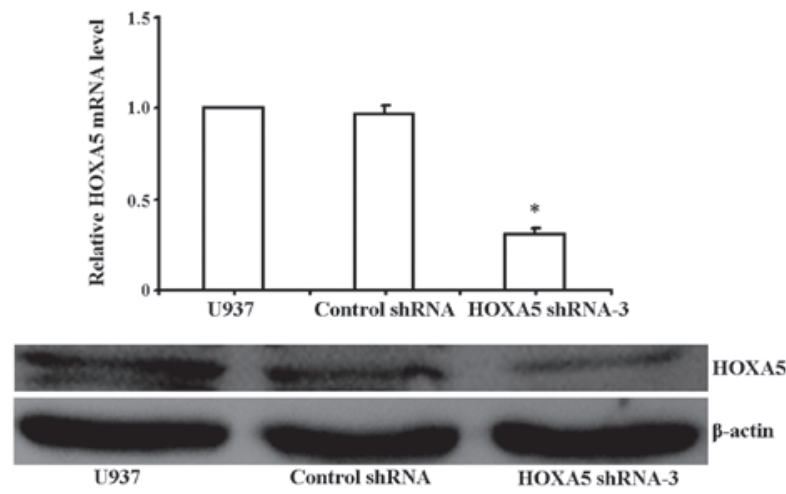

Figure 2. Effects of HOXA5 knockdown on HOXA5 mRNA and protein expression levels in U937 cells. The results of the (A) reverse transcription-quantitative polymerase chain reaction and the (B) western blot analyses demonstrated that $48 \mathrm{~h}$ post-transfection, HOXA5 shRNA-3 was determined to be the most effective for HOXA5 knockdown, compared with the parental U937 cells. "P<0.05 vs. parental U937 cells. HOXA5 (C) mRNA and (D) protein expression levels were measured with reverse transcription-quantitative polymerase chain reaction and western blot analyses. The HOXA5 mRNA and protein expression levels were significantly suppressed by the shRNA vector, compared with the control shRNA and parental U937 cells. ${ }^{*} \mathrm{P}<0.05$ vs. control shRNA and parental U937 cells. The average signal intensity was standardized to $\beta$-actin, and the data are presented as the mean \pm standard deviation of triplicate experiments. HOXA5, homeobox A5; shRNA, short hairpin RNA.

measured at $450 \mathrm{~nm}$ using an ELISA reader (F-7000; Hitachi High-Technologies Corporation, Tokyo, Japan).

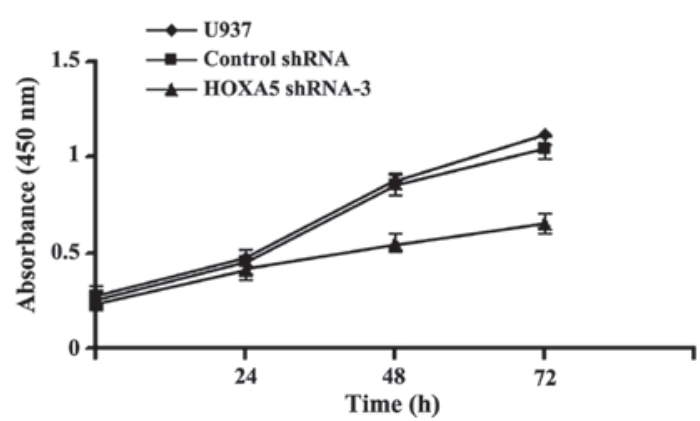

Figure 3. Effects of HOXA5 knockdown on U937 cell growth. Growth curves of U937 cells based on the results obtained from the Cell Counting kit- 8 assays in normal U937 cells and U937 cells transfected with control shRNA or HOXA5 shRNA-3. Transfection of U937 cells with HOXA5-targeted shRNA significantly decreased the levels of cell proliferation. The data are presented as the mean \pm standard deviation of triplicate experiments ${ }^{*} \mathrm{P}<0.05$. HOXA5, homeobox A5; shRNA, short hairpin RNA.

Cell cycle analysis. Cell cycle analysis was conducted using a Cell Cycle Detection kit (Nanjing KeyGEN Biotech Co., Ltd.) following the manufacturer's instructions $48 \mathrm{~h}$ post-transfection. Briefly, the cells were collected and fixed with $70 \%$ cold ethanol at $4^{\circ} \mathrm{C}$ overnight. The DNA was stained with RNase and propidium iodide (PI) for $30 \mathrm{~min}$ at room temperature, and then analyzed by flow cytometry (FACS FC500; Beckman Coulter).

Cell apoptosis analysis. A total of $1 \times 10^{5}$ cells were collected $48 \mathrm{~h}$ post-transfection, washed twice with PBS, and then resuspended in binding buffer (Nanjing KeyGEN Biotech Co., Ltd.). Cell suspension was stained with Annexin V-fluorescein isothiocyanate and PI (Nanjing KeyGEN Biotech Co., Ltd.) for 5-15 min at room temperature, and analyzed by flow cytometry.

Statistical analysis. The data are expressed as the mean \pm standard deviation. The results were evaluated by one-way analysis of variance using SPSS 16.0 software (SPSS, Inc., Chicago, IL, USA). $\mathrm{P}<0.05$ was considered to indicate a statistically significant result. 


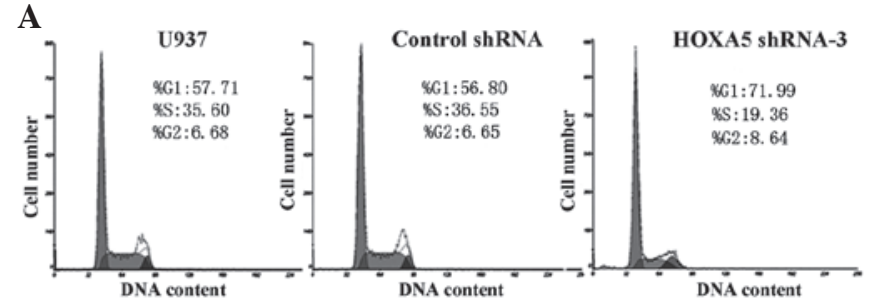

C

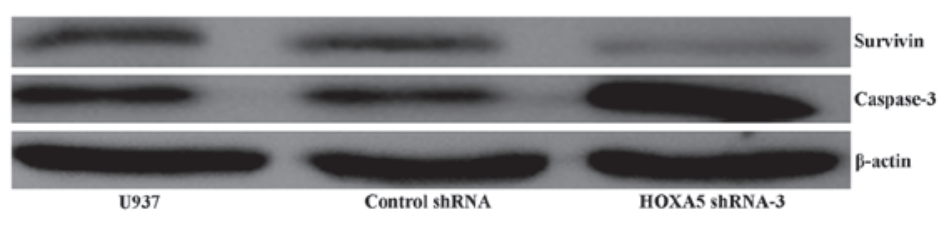

B
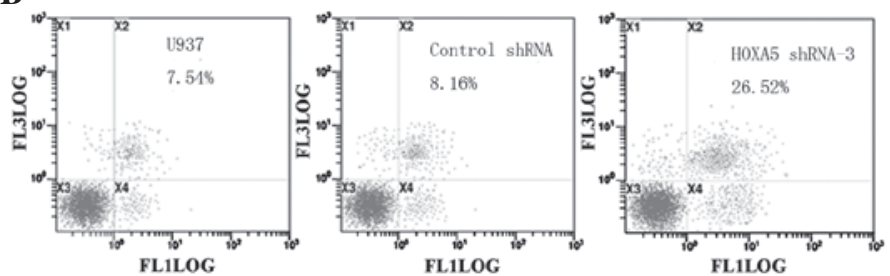

D

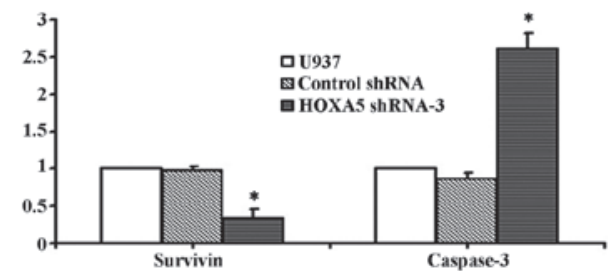

Figure 4. Effects of HOXA5 knockdown on U937 cell cycle distribution and cell apoptosis. (A) Changes in the cell cycle distribution of U937 cells following treatment with HOXA5 RNAi, as determined by flow cytometry. Following HOXA5 RNAi, flow cytometric analysis demonstrated an increase in the proportion of cells in the $\mathrm{G}_{0} / \mathrm{G}_{1}$ phase, and a decrease in the proportion of cells in the S phase in the HOXA5 shRNA groups, as compared with the controls. (B) Cell apoptosis was determined by Annexin V-fluorescein isothiocyanate/propidium iodide staining using a flow cytometer $48 \mathrm{~h}$ post-transfection. HOXA5 shRNA transfection significantly increased the apoptosis rates of the U937 cells. (C) The expression levels of apoptosis-associated proteins including survivin and caspase-3 were determined by western blot analysis. (D) The average signal intensity was standardized to $\beta$-actin, and the data are presented as the mean \pm standard deviation of triplicate experiments "P<0.05 vs. control shRNA and parental U937 cells. RNAi, RNA interference; HOXA5, homeobox 5A; shRNA, short hairpin RNA.

A

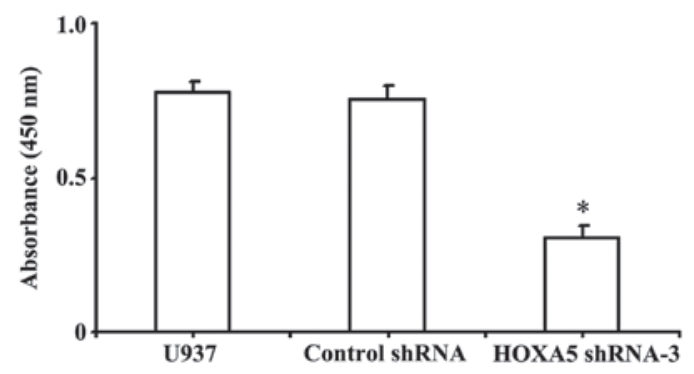

B

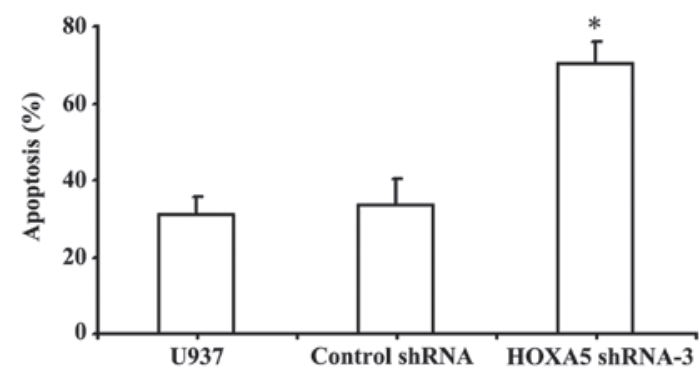

Figure 5. Effects of HOXA5 knockdown on the chemosensitivity of U937 cells to cytarabine. (A) Cell viability following treatment with cytarabine $(50 \mathrm{ng} / \mathrm{ml}, 48 \mathrm{~h})$ was measured using a Cell Counting kit-8 assay. The data are shown as the mean \pm standard deviation of triplicate experiments. (B) Cell apoptosis following treatment with cytarabine $(50 \mathrm{ng} / \mathrm{ml}, 48 \mathrm{~h}$ ) was determined with fluorescence-activated cell sorting analysis. The data are presented as the mean \pm standard deviation of triplicate experiments "P $<0.05$ vs. control shRNA and parental U937 cells.. HOXA5, homeobox 5A; shRNA, short hairpin RNA.

\section{Results}

Efficacy of shRNA vectors in decreasing HOXA5 expression in U937 cells. A green fluorescent protein (GFP)-containing vector, pGPH1, was used to knock down HOXA5. Post-transfection, $>70 \%$ of cells were GFP-positive, indicating high transfection efficiency (Fig. 1). To evaluate the effects of shRNAs on gene silencing, three HOXA5 shRNAs targeting the HOXA5 gene (HOXA5 shRNA-1, 2 and 3) were investigated in U937 cells by RT-qPCR and western blotting, with U937 cells alone serving as a control. HOXA5 shRNA-3 was determined to be the most effective compared with the other shRNAs. shRNA-3 induced a marked downregulation of HOXA5 transcript in U937 cells after 48 h (Fig. 2A and B; $\mathrm{P}<0.05)$. RT-qPCR analysis revealed that HOXA5 mRNA expression levels in the U937 cells transfected with HOXA5 shRNA-3 decreased by $\sim 70 \%$, compared with those of the untransfected U937 cells, whereas the control shRNA had no influence on HOXA5 mRNA expression levels in the U937 cells (Fig. 2C; $\mathrm{P}<0.05$ ). Western blot analysis also showed a significant decrease in HOXA5 expression in the U937 cell lines (Fig. 2D; $\mathrm{P}<0.05$ ).

Knockdown of HOXA5 expression inhibits cell proliferation as determined by a CCK- 8 assay. To determine whether downregulation of HOXA5 affected cell growth in vitro, the levels of cell proliferation were assessed following HOXA5 RNAi by CCK-8 assay, and the results are shown in Fig. 3. After $0,24,48$ and $72 \mathrm{~h}$ transfection, the growth of HOXA5 shRNA-3 transfected cells decreased compared with cells transfected with control shRNA and parental U937 $(\mathrm{P}<0.05)$, suggesting that transfection with HOXA5 shRNA-3 inhibited cell proliferation.

Knockdown of HOXA5 expression induces cell cycle $G_{1}$ phase arrest in $U 937$ cells in vitro. To evaluate the effects of HOXA5 knockdown on cell cycle distribution in U937 cells, DNA content was measured by flow cytometry in HOXA5 shRNA-3 transfected cells and control cells. As shown in Fig. 4A, $48 \mathrm{~h}$ post-transfection, interference of HOXA5 expression led to cell cycle arrest in the $\mathrm{G}_{1}$ phase, compared with the controls $(P<0.05)$. In addition, the increase in the $G_{1}$ phase cell 
population was accompanied by a concomitant decrease in cells in the S phase in the U937 cell lines with HOXA5 knockdown $(\mathrm{P}<0.05)$, without any observable changes in the cell population in the $G_{2}$ phase. These data suggest that the induction of $\mathrm{G}_{1}$ phase arrest accounts for the inhibitory effects of HOXA5 knockdown on U937 cell growth.

Knockdown of HOXA5 induces U937 cell apoptosis. The effects of HOXA5 knockdown on cell apoptosis were also investigated using flow cytometric analysis. Apoptotic rates were significantly higher in HOXA5 shRNA-3-transfected U937 cells. As shown in Fig. 4B, U937 cells transfected with HOXA5 shRNA-3 underwent increased apoptosis, compared with control shRNA and parental U937 cells. Furthermore, western blot analysis demonstrated that compared with control cells, the protein expression levels of survivin were significantly reduced, whereas the expression levels of caspase-3 were significantly increased in HOXA5 shRNA-3-transfected U937 cells (Fig. 4C; $\mathrm{P}<0.05$ ). These results suggest that inhibition of cell growth by HOXA5 shRNA-3 induces cell death in U937 cells via apoptotic events.

Knockdown of HOXA5 increases the sensitivity of U937 cells to cytarabine. To assess whether downregulation of HOXA5 was able to enhance the sensitivity of leukemic cells to cytarabine, a common antitumor drug used in the treatment of AML, 48 h post-transfection HOXA5 shRNA-3-transfected cells, control shRNA-transfected cells, and parental U937 cells were incubated with cytarabine for $24 \mathrm{~h}$, and cell proliferation inhibition was determined by a CCK-8 assay. As shown in Fig. 5A, compared with the controls, HOXA5 shRNA-3 exhibited significantly higher inhibitory levels on cell proliferation, indicating enhanced sensitivity to cytarabine $(\mathrm{P}<0.05)$. Furthermore, the present study investigated whether knockdown of HOXA5 was able to enhance cytarabine-induced cell apoptosis. HOXA5 shRNA-3 transfection combined with cytarabine resulted in a significant increase in apoptotic cell death, compared with cytarabine or HOXA5 shRNA-3 alone (Fig. 5B; $\mathrm{P}<0.05$ ), suggesting that HOXA5 knockdown was able to enhance the sensitivity of U937 cells to cytarabine.

\section{Discussion}

Deregulation of HOX genes influences tumorigenesis and cancer cell biology via differentiation, apoptosis, receptor signaling and other unknown mechanisms $(15,16)$. Aberrant expression of Hox has been observed in various types of cancer, such as hematologic malignancies, breast carcinoma, prostate cancer and lung cancer (17-21). Previous studies have suggested that overexpression of individual HOXA genes alone or in combination with MEIS1 results in leukemia (22-24). HOXA5 is a member of the HOX gene family and its overexpression in hematopoietic progenitor cells results in increased granulocytic/monocytic differentiation, but reduced erythroid/megakaryocytic differentiation (9). HOXA5 is critical for leukemic transformation in CALM-AF10-mediated leukemia, and is overexpressed in numerous leukemia cell lines $(25,26)$. These results suggested that HOXA5 is an important regulator of hematopoietic lineage determination and maturation. Therefore, the present study was designed to investigate the cellular functions of HOXA5, in order to elucidate the mechanism underlying its contribution to leukemia.

The present study used shRNA to reduce the expression of HOXA5 in human U937 leukemia cell lines. The selected shRNA-containing vector efficiently suppressed HOXA5 expression at the mRNA and protein levels. The effect of HOXA5 knockdown on the cellular functions of U937 cells was subsequently explored. Cell proliferation of U937 cells transfected with HOXA5 shRNA-3 also indicated that the cell growth of U937 cells decreased significantly compared with control cells. Cell cycle analysis demonstrated that the percentage of cells in the $G_{1}$ phase was markedly increased, and those in the $\mathrm{S}$ phase was markedly decreased in HOXA5 shRNA-3-transfected U937 cells. These results suggested that knockdown of the HOXA5 gene was able to arrest cell cycle progression, and inhibit cell proliferation. A flow cytometric apoptosis assay demonstrated that HOXA5-specific shRNA treatment resulted in a significant increase in the percentage of cell apoptosis.

Apoptosis is regulated by a balance of pro-apoptotic and anti-apoptotic genes, in which multiple genes and signaling pathways are implicated. Abnormal expression and functional changes of these genes are closely associated with tumorogenesis. Survivin is a member of the inhibitor of apoptosis family. The survivin protein is able to inhibit caspase activation via intrinsic and extrinsic signaling pathways, thereby leading to negative regulation of apoptosis or programmed cell death (27). These results have been demonstrated by the disruption of survivin induction signaling pathways leading to increased levels of apoptosis and a decrease in tumor growth (28-32). Caspase-3 is an important enzyme in apoptosis and the executor of apoptosis. The two signaling pathways of cellular apoptosis, the intrinsic and extrinsic signaling pathways, converge at caspase-3 to activate other caspases leading to a proteolytic cascade and eventually apoptotic morphology. Western blot analysis demonstrated that transfection with HOXA5-targeted shRNA significantly decreased the expression levels of survivin, and elevated the expression levels of caspase-3 compared with the controls, implying a role for HOXA5 as a regulator of the expression of apoptosis-associated molecules.

Cytarabine is a DNA polymerase inhibitor widely used for the treatment of several types of malignancies, including leukemia. The present study demonstrated that HOXA5 knockdown significantly enhances the cytotoxicity of cytarabine, and the combination of cytarabine and HOXA5-targeted shRNA exerts greater antiproliferative effects compared with cytarabine treatment alone, demonstrating the role of HOXA5 in the chemoresistance of U937 cells. Therefore, these results suggested that suppression of HOXA5 by shRNA may sensitize leukemic cells to cytarabine. These findings highlight the potential of combining conventional chemotherapeutics with gene therapy as an attractive therapeutic strategy for leukemia.

In conclusion, the results of the present study demonstrated that HOXA5 knockdown with shRNA in U937 human leukemia cells exerted antiproliferative effects, induced apoptosis and cell cycle arrest, and enhanced the cytotoxicity of cytarabine. These findings suggested that the shRNA-silencing of HOXA5 may be considered as a promising therapeutic target 
for leukemia, and may be used with conventional chemotherapeutics to increase therapeutic efficacy.

\section{Acknowledgements}

The authors of the present study would like to thank Dr Xie Shuyang and Dr Li Youjie for their technical assistance. The present study was supported by funding from the NCET-10-0919 'Taishan Scholar' position of the National Natural Science Foundation (grant nos. 31371321 and 81200601); the Shandong Science and Technology Committee (grant no. 2010GSF10264); and the Foundation of Shandong Educational Committee of China (grant nos. J10LC60 and J11LC01).

\section{References}

1. Passegué E, Jamieson CH, Ailles LE and Weissman IL: Normal and leukemic hematopoiesis: Are leukemias a stem cell disorder or a reacquisition of stem cell characteristics? Proc Natl Acad Sci USA 100 (Suppl 1):11842-11849, 2003.

2. Jemal A, Siegel R, Xu J and Ward E: Cancer statistics. 2010. CA Cancer J Clin 60: 277-300, 2010.

3. Estey E and Döhner H: Acute myeloid leukaemia. Lancet 368: 1894-1907, 2006.

4. Bali P, George P, Cohen P, Tao J, Guo F, Sigua C, Vishvanath A, Scuto A, Annavarapu S, Fiskus W, et al: Superior activity of the combination of histone deacetylase inhibitor LAQ824 and the FLT-3 kinase inhibitor PKC412 against human acute myelogenous leukemia cells with mutant FLT-3. Clin Cancer Res 10: 4991-4997, 2004.

5. Christiansen DH, Andersen MK, Desta F and Pedersen-Bjergaard J: Mutations of genes in the receptor tyrosine kinase (RTK)/RAS-BRAF signal transduction pathway in therapy-related myelodysplasia and acute myeloid leukemia. Leukemia 19: 2232-2240, 2005.

6. Plass C, Oakes C, Blum W and Marcucci G: Epigenetics in acute myeloid leukemia. Semin Oncol 35: 378-387, 2008.

7. Yao K, Xing H, Yang W, Liao A, Wu B, Li Y, Zhang R and Liu Z. Knockdown of RLIP76 expression by RNA interference inhibits proliferation, enhances apoptosis, and increases chemosensitivity to daunorubicin in U937 leukemia cells. Tumour Biol 35 : 8023-8031, 2014.

8. Cillo C, Cantile M, Faiella A and Boncinelli E: Homeobox genes in normal and malignant cells. J Cell Physiol 188: 161-169, 2001.

9. Bullinger L, Döhner K, Bair E, Fröhling S, Schlenk RF, Tibshirani R, Döhner H and Pollack JR: Use of gene-expression profiling to identify prognostic subclasses in adult acute myeloid leukemia. N Engl J Med 350: 1605-1616, 2004.

10. Grubach L, Juhl-Christensen C, Rethmeier A, Olesen LH, Aggerholm A, Hokland P and Ostergaard M: Gene expression profiling of Polycomb: Hox and Meis genes in patients with acute myeloid leukaemia. Eur J Haematol 81: 112-122, 2008.

11. Thorsteinsdottir U, Sauvageau G and Humphries RK. Hox homeobox genes as regulators of normal and leukemic hematopoiesis. Hematol Oncol Clin North Am 11: 1221-1237, 1997.

12. Crooks GM, Fuller J, Petersen D, Izadi P, Malik P, Pattengale PK, Kohn DB and Gasson JC: Constitutive HOXA5 expression inhibits erythropoiesis and increases myelopoiesis from human hematopoietic progenitors. Blood 94: 519-528, 1999.

13. Lawrence HJ and Largman C: Homeobox genes in normal hematopoiesis and leukemia. Blood 80: 2445-2453, 1992.
14. Celetti A, Barba P, Cillo C, Rotoli B, Boncinelli E and Magli MC. Characteristic patterns of HOX gene expression in different types of human leukemia. Int J Cancer 237-244, 1993.

15. Drabkin HA, Parsy C, Ferguson K, Guilhot F, Lacotte L, Roy L, Zeng C, Baron A, Hunger SP, Varella-Garcia M, et al: Quantitative HOX expression in chromosomally defined subsets of acute myelogenousleukemia. Leukemia 16: 186-195, 2002.

16. Golub TR, Slonim DK, Tamayo P, Huard C, Gaasenbeek M, Mesirov JP, Coller H, Loh ML, Downing JR, Caligiuri MA, Bloomfield CD and Lander ES. Molecular classification of cancer: Class discovery and class prediction by gene expression monitoring. Science 286: 531-537, 1999.

17. Shah N and Sukumar S: The hox genes and their roles in oncogenesis. Nat Rev Cancer 10: 361-371, 2010.

18. Graham A, Papalopulu N and Krumlauf R: The murine and drosophila homeobox gene complexes have common features of organization and expression. Cell 57: 367-378, 1989.

19. Waltregny D, Alami Y, Clausse N, de Leval J and Castronovo V: Overexpression of the homeobox gene HOXC8 in human prostate cancer correlates with loss of tumor differentiation. Prostate 50: 162-169, 2002.

20. Makiyama K, Hamada J, Takada M, Murakawa K, Takahashi Y, Tada M, Tamoto E, Shindo G, Matsunaga A, Teramoto K, et al: Aberrant expression of HOX genes in human invasive breast carcinoma. Oncol Rep 13: 673-679, 2005.

21. Abe M, Hamada J, Takahashi O, Takahashi Y, Tada M, Miyamoto M, Morikawa T, Kondo S and Moriuchi T: Disordered expression of HOX genes in human non-small cell lung cancer. Oncol Rep 15: 797-802, 2006.

22. Thorsteinsdottir U, Mamo A, Kroon E, Jerome L, Bijl J, Lawrence HJ, Humphries K and Sauvageau G: Overexpression of the myeloid leukemia-associated hoxa9 gene in bone marrow cells induces stem cell expansion. Blood 99: 121-129, 2002.

23. Bach C, Buhl S, Mueller D, García-Cuéllar MP, Maethner E and Slany RK: Leukemogenic transformation by HOXA cluster genes. Blood 115: 2910-2918, 2010.

24. Mulgrew NM, Kettyle LM, Ramsey JM, Cull S, Smyth LJ, Mervyn DM, Bijl JJ and Thompson A: c-Met inhibition in a HOXA9/Meis1 model of CN-AML. Dev Dyn 243: 172-181, 2014.

25. Okada Y, Jiang Q, Lemieux M, Jeannotte L, Su L and Zhang Y: Leukaemic transformation by CALM-AF10 involves upregulation of hoxa5 by hDOT1 L. Nat Cell Biol 8: 1017-1024, 2006.

26. Quentmeier H, Dirks WG, Macleod RA, Reinhardt J, Zaborski M and Drexler HG: Expression of HOX genes in acute leukemia cell lines with and without MLL translocations. Leuk Lymphoma 45: 567-574, 2004.

27. Deveraux QL and Reed JC: IAP family proteins-suppressors of apoptosis. Gen Dev 13: 239-252, 1999.

28. Karami H, Baradaran B, Esfahani A, Estiar MA, Naghavi-Behzad M, Sakhinia M and Sakhinia E: siRNA-mediated silencing of survivin inhibits proliferation and enhances etoposide chemosensitivity in acute myeloid leukemia cells. Asian Pac J Cancer Prev 14: 7719-7724, 2013.

29. Riedl SJ and Shi Y: Molecular mechanisms of caspase regulation during apoptosis. Nat Rev Mol Cell Biol 5: 897-907, 2004.

30. Sommer KW, Schamberger CJ, Schmidt GE, Sasgary S and Cerni C: Inhibitor of apoptosis protein (IAP) survivin is upregulated by oncogenic c-H-Ras. Oncogene 22: 4266-4280, 2003.

31. Yamanaka K, Nakata M, Kaneko N, Fushiki H, Kita A, Nakahara T, Koutoku H and Sasamata M: YM155, a selective survivin suppressant, inhibits tumor spread and prolongs survival in a spontaneous metastatic model of human triple negative breast cancer. Int J Oncol 39: 569-575, 2011.

32. Blanc-Brude OP, Mesri M, Wall NR, Plescia J, Dohi T and Altieri DC: Therapeutic targeting of the survivin pathway in cancer: Initiation of mitochondrial apoptosis and suppression of tumor-associated angiogenesis. Clin Cancer Res 9: 2683-2692, 2003. 